\title{
Enhanced Recovery in Gynecologic Surgery
}

\author{
Eleftheria Kalogera, MD, Jamie N. Bakkum-Gamez, MD, Christopher J. Jankowski, MD, \\ Emanuel Trabuco, MD, Jenna K. Lovely, DrPH, Sarah Dhanorker, Pamela L. Grubbs, RN, \\ CNS, Amy L. Weaver, Lindsey R. Haas, Bijan J. Borah, PhD, April A. Bursiek, RN, Michael \\ T. Walsh, MD, William A. Cliby, MD, and Sean C. Dowdy, MD \\ Division of Gynecologic Surgery, the Department of Anesthesiology, Hospital Pharmacy Services, \\ the Department of Nursing, the Division of Biomedical Statistics and Informatics, and the \\ Department of Health Sciences, Division of Health Care Policy and Research, Mayo Clinic, \\ Rochester, Minnesota.
}

\section{Abstract}

OBJECTIVE-To investigate the effects of enhanced recovery (a multimodal perioperative care enhancement protocol) in patients undergoing gynecologic surgery.

METHODS-Consecutive patients managed under an enhanced recovery pathway and undergoing cytoreduction, surgical staging, or pelvic organ prolapse surgery between June 20, 2011, and December 20, 2011, were compared with consecutive historical controls (March to December 2010) matched by procedure. Wilcoxon rank-sum, $\chi^{2}$, and Fisher's exact tests were used for comparisons. Direct medical costs incurred in the first 30 days were obtained from the Olmsted County Healthcare Expenditure and Utilization Database and standardized to 2011 Medicare dollars.

RESULTS-A total of 241 enhanced recovery women in the case group (81 cytoreduction, 84 staging, and 76 vaginal surgery) were compared with women in the control groups. In the cytoreductive group, patient-controlled anesthesia use decreased from $98.7 \%$ to $33.3 \%$ and overall opioid use decreased by $80 \%$ in the first 48 hours with no change in pain scores. Enhanced recovery resulted in a 4-day reduction in hospital stay with stable readmission rates (25.9\% of women in the case group compared with $17.9 \%$ of women in the control group) and 30-day cost savings of more than $\$ 7,600$ per patient (18.8\% reduction). No differences were observed in rate (63\% compared with $71.8 \%$ ) or severity of postoperative complications (grade 3 or more: $21 \%$ compared with 20.5\%). Similar, albeit less dramatic, improvements were observed in the other two cohorts. Ninety-five percent of patients rated satisfaction with perioperative care as excellent or very good.

CONCLUSIONS-Implementation of enhanced recovery was associated with acceptable pain management with reduced opioids, reduced length of stay with stable readmission and morbidity rates, good patient satisfaction, and substantial cost reductions.

Over the previous decade, important progress has been achieved in both benign and oncologic gynecologic surgery, including further refinement of minimally invasive surgery,

(C) 2013 by The American College of Obstetricians and Gynecologists. Published by Lippincott Williams \& Wilkins

Corresponding author: Sean C. Dowdy, MD, $2001^{\text {st }}$ Street SW, Rochester, MN 55905; dowdy.sean@ mayo.edu..

The authors thank Drs. David Larson and Robert Cima from Colorectal Surgery who pioneered enhanced recovery at the Mayo Clinic, Rochester and served as important consultants throughout this project.

Presented at the 14tah Biennial meeting of the International Gynecologic Cancer Society, October 13-16, 2012, Vancouver, Canada.

Financial Disclosure

The authors did not report any potential conflicts of interest. 
introduction of the sentinel lymph node concept, individualized lymphadenectomy for endometrial cancer, and adoption of optimal cytoreduction to no visible residual disease for patients with ovarian cancer. ${ }^{1-3}$ These practice changes have reduced surgical morbidity, shortened recovery, and improved oncologic outcomes. ${ }^{1-3}$ However, little attention has been given to the optimization of perioperative care. Few data exist to support traditional components of perioperative care including preoperative bowel preparation, prolonged fasting and use of nasogastric tubes, intraabdominal drains, bed rest, and gradual introduction of oral feeding. Longer length of hospital stay has been correlated with lower quality of life, and health care institutions are subject to increasing pressures to reduce costs. ${ }^{4}$

A number of management pathways has been introduced primarily in colorectal surgery to hasten recovery and attenuate the stress response associated with surgery. ${ }^{5,6}$ Key elements common to all enhanced recovery pathways (a term coined by Dr. Kehlet ${ }^{5,6}$ ) include: preoperative patient education, reduction of preoperative fasting, omission of bowel preparation, perioperative normovolemia, limited use of nasogastric tubes and drains, early removal of urinary catheters, aggressive multimodal analgesia to minimize opiate consumption, early postoperative mobilization, prokinetics to enhance gastrointestinal motility, and early enteral nutrition. A growing body of evidence outside of gynecology including randomized controlled trials ${ }^{7-10}$ has shown that enhanced recovery speeds convalescence and reduces morbidity and cost while maintaining patient satisfaction and quality of life..$^{9,11-13}$

Few groups have tested enhanced recovery in gynecologic surgery, and most cohorts were small and included only patients with benign disorders. ${ }^{14-20}$ The purpose of this investigation was to examine the effect of our enhanced recovery pathway on hospital length of stay, morbidity, cost, and patient satisfaction for women undergoing major abdominal surgery for gynecologic malignancies and vaginal reconstructive procedures for pelvic organ prolapse.

\section{METHODS}

A multimodal enhanced recovery pathway was developed by a multidisciplinary team of anesthesiologists, gynecologic oncologists, urogynecologists, pharmacists, and nursing staff based on the most current recommendations from the literature but accounting for the unique issues presented by gynecologic surgical patients (Box 1). Enhanced recovery was instituted on June 20,2011, as a quality improvement practice change and was used for all inpatients treated in the Division of Gynecologic Surgery, Mayo Clinic, Rochester, Minnesota, from that date forward. Three cohorts were analyzed: 1) staging laparotomy for gynecologic malignancies including hysterectomy, lymphadenectomy and omentectomy only; 2) complex cytoreductive surgery cases, which included patients described in cohort 2 in addition to bowel resection, splenectomy, diaphragmatic resection, extensive cytoreduction, or all of these; and 3) urogynecologic pelvic organ prolapse surgery including posthysterectomy pelvic floor repair or vaginal hysterectomy with concomitant repairs (isolated hysterectomies were not included); the latter group of procedures will be referred to in the article as vaginal surgery cases.

This was a retrospective cohort study. The power calculation was performed based on our main outcome, reduction in hospital length of stay. To achieve $80 \%$ power to detect a statistically significant 2-day reduction in length of stay in the staging cohort as well as the complex cytoreductive cohort, a total of 39 patients were needed in each cohort; 15 patients were necessary in the pelvic organ prolapse cohort to detect a 1-day reduction in length of stay. Consecutive patients within these three cohorts were prospectively identified from June 
20, 2011, to December 20, 2011, and followed for at least 30 days. Enhanced recovery patients were then compared with consecutive historic controls matched one to one by procedure type from March 1, 2010, to December 23, 2010, before enhanced recovery was implemented. The only exclusion criterion was lack of research authorization. Of note, no significant changes in technology, surgical techniques, or surgical teams took place between the study period and the period from which historic controls were drawn. Before enhanced recovery, postoperative management was not standardized among the seven gynecologic oncologists and four urogynecologists at our institution. However, bowel preparations, caloric restriction, intraoperative hypervolemia, use of patient-controlled analgesia, and the use of surgical drains and catheters were routine practices. Local wound infiltration, triple antiemetics, and prokinetics were generally not used in historical controls. An interim analysis was performed at 3 months to confirm that this practice change did not increase complication rates.

Relevant data were abstracted from our electronic medical records (including outside medical records when necessary). For hospital length of stay, the day of surgery was defined as postoperative day 0 . Total length of stay was calculated by adding readmission length of stay (if readmission occurred) to primary hospitalization length of stay. Postoperative hypotension was defined as a $10 \%$ decrease from the preoperative mean arterial pressure. Opioid use was quantified using oral morphine equivalents. ${ }^{21}$ "Opioid tolerance" was defined as at least $60 \mathrm{mg}$ oral morphine equivalents per day. The efficacy of pain control was assessed using pain scores on a scale of $1-10$. The Accordion severity grading system was used to measure severity of postoperative complications; grade 3 and higher complications were considered severe. ${ }^{22}$ Return of gastrointestinal function was defined as positive flatus or bowel movement. Patients were discharged when all of the following criteria were met: pain controlled with oral medications alone, tolerating solid food without intravenous hydration, independently ambulatory, and no suspicion of a complication. Confidential patient satisfaction surveys were administered by nonstudy providers to all patients before dismissal as part of a continuous assessment of our surgical inpatient unit.

Cost data for the study patients were captured from Olmsted County Healthcare Expenditure and Utilization Database, a research database jointly funded by Mayo Clinic's Division of Health Care Policy and Research and the National Institutes of Health-funded Rochester Epidemiology Project. ${ }^{23}$ This unique database provides standardized inflation-adjusted estimate of costs in 2011 constant dollars (regardless of payer or plan) for every service and procedure received by patients seen at the Mayo Clinic. However, the costs of outpatient pharmacy services and nursing home care were not captured in the Olmsted County Healthcare Expenditure and Utilization Database.

This investigation was reviewed by the Mayo Foundation institutional review board and as a quality improvement project was considered exempt from institutional review board review for initial implementation (45 CFR 46.101, item 4); however, institutional review board approval was obtained retrospectively for publication of findings. In accordance with the Minnesota Statute for Use of Medical Information in Research, only those patients who consented to the use of their medical records were included in final analysis and publication.

Categorical variables were summarized using actual counts (\%) and continuous variables using mean (standard deviation) or median (interquartile range) as appropriate. The $\chi^{2}$ test or the Fisher's exact test, as appropriate when an expected cell count in a contingency table was less than five, were used to compare categorical variables and the Wilcoxon rank-sum test for continuous variables. Cost analysis included all-cause 30-day postsurgical costs. The difference in median cost between groups was tested using a Wilcoxon rank-sum test. $P>.05$ 
was considered statistically significant for all statistical comparisons. Statistical analyses were performed using the SAS 9.2 software package.

\section{RESULTS}

A total of 241 enhanced recovery cases ( 81 complex cytoreductive, 84 staging, and 76 vaginal surgery cases) and 235 historic controls (78 complex cytoreductive, 80 staging, and 77 vaginal cases) met criteria for inclusion in the study analyses (after 14 patients, four women in the case group and 10 women in the control group, were excluded as a result of lack of research consent).

In the complex cytoreductive cohort, patient baseline, operative, and postoperative characteristics are shown in Table 1. Clinically significant differences between women in the case group and women in the control group were limited to a higher proportion of clean wounds and "opioid-tolerant" patients in the enhanced recovery cohort.

Compliance with enhanced recovery pathway is shown in Appendix 1 (available online at http://links.lww.com/AOG/A405). The lower than expected rate of preload carbohydrate drink administration was the result of a diminished supply of the product at our pharmacies (the use of this product has since been discontinued as a result of cost considerations). The rate of preoperative celecoxib and postoperative nonsteroidal anti-inflammatory drug administration is reflective of the fact that these medications were omitted in patients with contraindications. The expected shift from a singledrug to multimodal postoperative nausea and vomiting prophylaxis protocol was observed with almost all enhanced recovery patients receiving at least two antiemetic medications intraoperatively.

Control patients received on average $1,059 \mathrm{~mL}$ more crystalloid intraoperatively compared with enhanced recovery patients $(P>.001)$. Despite administration of less crystalloid, there was no increase in the frequency or duration of hypotension intraoperatively $(P=.25)$.

Women in the case group received significantly less opioids (80\% reduction in first 48 hours after return to room) with an increase in the use of scheduled nonsteroidal anti-inflammatory drugs, acetaminophen, and tramadol (Appendix 2, available online at http://links.lww.com/ AOG/A406). Patient-controlled analgesia was infrequently required in the women in the case group compared with the historic controls (women in the enhance recovery group: 27 [33\%] compared with women in the historic control group:77 [98.7\%], $P>.001$ ) (Appendix 1, http://links.lww.com/AOG/A405). Despite a significant reduction in opioid and patientcontrolled analgesia use, pain scores were unchanged in the women in the case group compared with women in the control group with the exception of an improvement at the time of return to room after postanesthesia care unit discharge (mean [standard deviation]: women in the case group 4.4 [2.3] compared with women in the control group 5.6 [2.6], $P=$. 003) (Appendix 3, available online at http://links.lww.com/AOG/A407).

Although a more aggressive postoperative nausea and vomiting protocol was adopted intraoperatively, more nausea and vomiting was observed in the enhanced recovery group with a significant increase on postoperative day 2 (women in the case group compared with women in the control group: nausea 45 of 81 [55.6\%] compared with 30 of 78 [38.5\%], $P=$. 031; vomiting 14 of 81 [17.3\%] compared with two of 78 [2.6\%], $P=.002$ ). Overall, the amount of antiemetic medication that was required to control patients' symptoms was not different between groups. Women in the case group had a 1-day earlier return of bowel function compared with the historic controls $(P>.001)$. The rate of postoperative ileus was no different between groups. 
Thirty-day rates of complications, readmission, mortality, and the severity of complications did not differ between groups. Introduction of enhanced recovery resulted in a 4-day reduction in the mean length of stay compared with historic controls $(P>.001)$. One-third of patients in the complex cytoreductive enhanced recovery group were discharged within 4 days of surgery compared with only $6.4 \%$ of women in the control group $(P>.001)$. The reduction in length of stay was accompanied by a 30-day total cost of care savings of more than $\$ 7,600$ per patient for this cohort, an $18.8 \%$ reduction (Table 2). Thus, more than $\$ 500,000$ in savings was achieved for just 81 patients $(P=.006)$.

In the staging cohort, patient baseline, operative, and postoperative characteristics were comparable to historic controls in all categories except median operative time, which was shorter by 30 minutes in the enhanced recovery group (median [interquartile range] 151 minutes [114-211] compared with 177 minutes [140-220], respectively; $P=.042$ ) (Table 1).

Like in the complex cytoreductive cohort, adherence to the protocol was good with regard to preoperative management, postoperative nausea and vomiting prophylaxis, perioperative fluid management, and a reduction in opioid use (Appendix 1, available online at http:// links.lww.com/AOG/A405). Patient-controlled analgesia was used 10 times less frequently in women in the case group compared with women in the control group (seven of 84 [8.3\%] compared with 78 of 80 [97.5\%], respectively; $P>.001$, Appendix 1 [available online at http://links.lww.com/AOG/A405]); nevertheless, a significant improvement in mean pain scores at the time of return to room was noted in women in the case group (4.3 compared with 5.2, respectively; $P=.030$ ). Women in the case group experienced significantly more nausea on the day of surgery after return to the room (28 of 84 [33.3\%] compared with 15 of 80 [18.8\%]; $P=.034)$. Similar to the complex cytoreductive group, 30-day outcomes remained unchanged after implementing enhanced recovery (Table 3 ).

Enhanced recovery resulted in a 2-day reduction in the mean length of stay for this cohort (Table 3). Length of stay was 3 days or less in $26.2 \%$ of women in the case group compared with 5\% of historical controls $(P>.001)$. Cost savings were more than $\$ 3,000$ per patient, although not statistically significant in this analysis (Table 2 ).

In the vaginal surgery cohort, there were no clinically significant differences between women in the case group and women in the control group with regard to patient baseline, operative, or postoperative characteristics (Table 1). Adherence to the protocol was very good with regard to preoperative management, nausea and vomiting prophylaxis, and postoperative pain management (Appendix 1, available online at http://links.lww.com/AOG/ A405). In contrast to the laparotomy cases, the intraoperative fluid management did not differ between groups given that conservative volumes of intraoperative fluids were administered during these cases at baseline. However, a significant reduction in the volume of crystalloids administered on postoperative day 0 was observed in the women in the case group compared with the women in the control group (median [interquartile range] $176 \mathrm{~mL}$ [0-310] compared with 575 [350-1,000], respectively; $P>.001$ ). Intrathecal anesthesia was used more commonly in the enhanced recovery cohort (31 of 76 [40.8\%] compared with eight of 77 [10.4\%]; $P>.001$ ). Similar to the previous two laparotomy cohorts, patientcontrolled analgesia was used much less commonly in the enhanced recovery cohort (seven of 76 [9.2\%] compared with 61 of 77 [79.2\%]; $P>.001$, Appendix 1 [available online at http://links.lww.com/AOG/A405]).

A significant improvement in the mean pain score from admission to the postanesthesia care unit up until 4 hours after return to the room was observed in the women in the case group (postanesthesia care unit: 1.9 compared with $3.3, P>.001$; return to room: 2.8 compared with $4.7, P>.001 ; 4$ hours after return to room: 2.9 compared with $3.9, P=.015)$ reflective of the 
effect of intrathecal anesthesia or analgesia. More than 4 hours after return to the room, mean pain scores were comparable between groups. Again, vomiting was more frequently observed in the enhanced recovery group on postoperative day 0 (eight of 76 [10.5\%] compared with one of 77 [1.3\%], respectively; $P=.015$ ). There were no differences in 30-day outcomes (Table 3). A significant reduction in mean length of stay was observed after implementing enhanced recovery; almost half $(46.1 \%)$ of the women in the case group were discharged the day after surgery compared with only $6.5 \%$ of women in the control group $(P>.001)$ (Table 3). Within the enhanced recovery cohort, patients who received intrathecal anesthesia had a significantly shorter length of stay than those who did not (median length of stay 2 days compared with 3 days; $P=.021$ ) with no difference in hospital costs (median hospital costs intrathecal $\$ 9,381.41$ compared with no intrathecal $\$ 9,973.54 ; P=.51$ ).

Patient satisfaction was high in all studied aspects of perioperative care including patient education, quality of care during hospitalization, the discharge process, and pain management with 90-99\% rating satisfaction as excellent or very good (Appendix 4, available online at http://links.lww.com/AOG/A408). Satisfaction with control of postoperative nausea and vomiting was rated as excellent or very good in $87 \%$. Patient satisfaction surveys were not available in historic controls.

\section{DISCUSSION}

Dogmatic interventions that adversely affect postoperative recovery include the use of bowel preparations, caloric restriction, intraoperative hypervolemia, excessive opioid use, prolonged immobilization, and the use of drains and catheters. Enhanced recovery challenges traditional perioperative paradigms and has been shown to hasten postoperative recovery in colorectal surgery. 7,8,12,13,24-26 Prior investigations of enhanced recovery have shown only preliminary evidence of benefit ${ }^{18,19}$ and data are very limited for patients with gynecologic malignancies. ${ }^{18,19}$ One investigation describes 19 cases over 8 years, ${ }^{18}$ whereas the other included 69 patients who underwent cytoreduction with a median operating time of only 2 hours. ${ }^{19}$ The current investigation provides data demonstrating that our enhanced recovery pathway is associated with more rapid recovery when implemented in patients undergoing major abdominal surgery for gynecologic malignancies or in patients undergoing pelvic organ prolapse surgery. Specifically, use of enhanced recovery resulted in earlier return of gastrointestinal function, stable pain scores despite significantly reduced opioid use, excellent patient satisfaction despite a 4-day reduction in length of hospital stay, 30 -day cost savings of more than $\$ 7,600$ per patient (19\% reduction), and stable complication and readmission rates. As suggested by Maessen et al, ${ }^{27}$ continuous monitoring of adherence to the protocol elements will be necessary to ensure that the desired outcomes persist beyond the initial study period.

We consider all interventions (or lack thereof) outlined in Box 1 as critical to success, and clinicians should not expect similar benefits in their own patients by simply adopting early feeding alone. However, a few interventions deserve particular mention. We believe that euvolemia is paramount to the improved clinical appearance of patients the day after surgery and for the improved outcomes in this investigation. ${ }^{28,29}$ Partnering with anesthesiology to achieve this was critical. In our investigation, perioperative fluid restriction did not increase the duration or frequency of hypotension within the first 48 hours after surgery nor did it increase the incidence of acute renal failure. It is worth highlighting that transient oliguria (up to 24 hours after surgery) with urine output as low as $20 \mathrm{~mL} / \mathrm{h}$ is a normal response to surgical stress and does not require intervention. ${ }^{30,31}$ On the other hand, avoidance of preoperative dehydration (eg, mechanical bowel preparation) and caloric restriction, the use of prokinetics, and initiation of early feeding all contributed to acceleration of gastrointestinal function. ${ }^{10,17,18,32}$ In our complex cytoreductive cohort, roughly $40 \%$ of 
women in the case group underwent large bowel resection with no measurable untoward effects relative to women in the control group with regard to anastomotic leaks and abscesses. Despite its merits, a recent review and meta-analysis concluded that early feeding is associated with increased postoperative nausea and vomiting ${ }^{32}$ and might explain the higher frequency of nausea and vomiting observed in our investigation despite aggressive multimodal postoperative nausea and vomiting prophylaxis. Despite this increase in nausea and vomiting, $87 \%$ rated their satisfaction with nausea and vomiting control as excellent or very good, suggesting that early feeding is overall well tolerated.

Epidural analgesia and patient-controlled analgesia have been used commonly in prior enhanced recovery trials. ${ }^{33,34}$ After significant debate, our investigative group elected to omit epidural analgesia in patients undergoing laparotomy given concerns about fluid shifts and hypotension in a patient population who commonly present with large cancer burdens, poor nutrition, and several liters of ascites, particularly when adopting a strategy of euvolemia intraoperatively. Excellent results were obtained even without the use of intrathecal analgesia. However, patients undergoing pelvic organ prolapse surgery appeared to derive significant benefit from intrathecal anesthesia, and in the future, an attempt will be made to increase use in our institution.

Weaknesses of this investigation include that it was not designed as a randomized clinical trial. Considering accumulating data on the success of enhanced recovery pathways in other surgical subspecialties, our group felt that this initiative was best executed as a quality improvement study. However, we used historical controls 6 months before adoption of this pathway such that changes in practice patterns, techniques, and staffing were minimal between groups. Sample size was powered to detect differences in hospital length of stay. Thus, although we found no changes in morbidity, mortality, or readmission between groups, this study may be underpowered to detect such differences. Lastly, given the large number of changes contained within the management pathway, it is impossible to determine which intervention had the greatest effect on recovery. However, all of the elements of this pathway have merit with respect to various end points, including patient comfort and satisfaction, even if they potentially do not affect cost or length of stay. Furthermore, there are virtually no costs required to implement this pathway.

In summary, institution of an enhanced recovery pathway in gynecologic surgery resulted in significant improvement of postoperative outcomes including earlier return of gastrointestinal function, excellent pain management with significantly reduced opioid requirements, decreased length of hospital stay, excellent patient satisfaction combined with substantial cost reductions while maintaining stable complication and readmission rates.

Wome undergoing the most complex oncologic procedures appear to have the most to gain from this management strategy. At present all women undergoing inpatient gynecologic surgery at Mayo Clinic, regardless of diagnosis, are managed under the enhanced recovery pathway.

\section{Acknowledgments}

Supported in part by the Mayo Clinic Comprehensive Cancer Center (P30 CA 15083; S.C.D.) and the Office of Women's Health Research Building Interdisciplinary Careers in Women's Health (BIRCWH award K12 HD065987; J.N.B.-G.).

\section{REFERENCES}

1. Dowdy SC, Borah BJ, Bakkum-Gamez JN, Weaver AL, McGree ME, Haas LR, et al. Prospective assessment of survival, morbidity, and cost associated with lymphadenectomy in lowrisk endometrial cancer. Gynecol Oncol. 2012; 127:5-10. [PubMed: 22771890] 
2. Bristow RE, Tomacruz RS, Armstrong DK, Trimble EL, Montz FJ. Survival effect of maximal cytoreductive surgery for advanced ovarian carcinoma during the platinum era: a meta-analysis. $\mathbf{J}$ Clin Oncol. 2002; 20:1248-59. [PubMed: 11870167]

3. Van der Zee AG, Oonk MH, De Hullu JA, Ansink AC, Vergote I, Verheijen RH, et al. Sentinel node dissection is safe in the treatment of early-stage vulvar cancer. J Clin Oncol. 2008; 26:884-9. [PubMed: 18281661]

4. Sharma A, Sharp DM, Walker LG, Monson JR. Predictors of early postoperative quality of life after elective resection for colorectal cancer. Ann Surg Oncol. 2007; 14:3435-42. [PubMed: 17896155]

5. Kehlet H. Multimodal approach to control postoperative pathophysiology and rehabilitation. Br J Anaesth. 1997; 78:606-17. [PubMed: 9175983]

6. Kehlet H, Wilmore DW. Multimodal strategies to improve surgical outcome. Am J Surg. 2002; 183:630-41. [PubMed: 12095591]

7. Anderson AD, McNaught CE, MacFie J, Tring I, Barker P, Mitchell CJ. Randomized clinical trial of multimodal optimization and standard perioperative surgical care. Br J Surg. 2003; 90:1497-504. [PubMed: 14648727]

8. Khoo CK, Vickery CJ, Forsyth N, Vinall NS, Eyre-Brook IA. A prospective randomized controlled trial of multimodal perioperative management protocol in patients undergoing elective colorectal resection for cancer. Ann Surg. 2007; 245:867-72. [PubMed: 17522511]

9. Adamina M, Kehlet H, Tomlinson GA, Senagore AJ, Delaney CP. Enhanced recovery pathways optimize health outcomes and resource utilization: a meta-analysis of randomized controlled trials in colorectal surgery. Surgery. 2011; 149:830-40. [PubMed: 21236454]

10. Spanjersberg WR, Reurings J, Keus F, van Laarhoven CJ. Fast track surgery versus conventional recovery strategies for colorectal surgery. The Cochrane Database of Systematic Reviews. 2011; (Issue 2) Art. No.: CD007635. DOI: 10.1002/14651858. CD007635.pub2.

11. Khan S, Wilson T, Ahmed J, Owais A, MacFie J. Quality of life and patient satisfaction with enhanced recovery protocols. Colorectal Dis. 2010; 12:1175-82. [PubMed: 19594603]

12. Lovely JK, Maxson PM, Jacob AK, Cima RR, Horlocker TT, Hebl JR, et al. Case-matched series of enhanced versus standard recovery pathway in minimally invasive colorectal surgery. Br J Surg. 2012; 99:120-6. [PubMed: 21948187]

13. Sammour T, Zargar-Shoshtari K, Bhat A, Kahokehr A, Hill AG. A programme of Enhanced Recovery After Surgery (ERAS) is a cost-effective intervention in elective colonic surgery. N Z Med J. 2010; 123:61-70. [PubMed: 20717178]

14. Ottesen M, Sorensen M, Kehlet H, Ottesen B. Short convalescence after vaginal prolapse surgery. Acta Obstet Gynecol Scand. 2003; 82:359-66. [PubMed: 12716321]

15. Ottesen M, Sorensen M, Rasmussen Y, Smidt-Jensen S, Kehlet H, Ottesen B. Fast track vaginal surgery. Acta Obstet Gynecol Scand. 2002; 81:138-46. [PubMed: 11942904]

16. Sjetne IS, Krogstad U, Odegard S, Engh ME. Improving quality by introducing enhanced recovery after surgery in a gynaecological department: consequences for ward nursing practice. Qual Saf Health Care. 2009; 18:236-40. [PubMed: 19468009]

17. Eberhart LHJ, Koch T, Ploger B, Wagner U, Wulf H, Zwiorek L, et al. Enhanced recovery after major gynaecological surgery for ovarian cancer-an objective and patient-based assessment of a traditional versus a multimodal 'fast track' rehabilitation programme. Anasthesiologie Und Intensivmedizin. 2008; 49:180-94.

18. Gerardi MA, Santillan A, Meisner B, Zahurak ML, Diaz Montes TP, Giuntoli RL II, et al. A clinical pathway for patients undergoing primary cytoreductive surgery with rectosigmoid colectomy for advanced ovarian and primary peritoneal cancers. Gynecol Oncol. 2008; 108:282-6. [PubMed: 18023851]

19. Marx C, Rasmussen T, Jakobsen DH, Ottosen C, Lundvall L, Ottesen B, et al. The effect of accelerated rehabilitation on recovery after surgery for ovarian malignancy. Acta Obstet Gynecol Scand. 2006; 85:488-92. [PubMed: 16612713]

20. Moller C, Kehlet H, Friland SG, Schouenborg LO, Lund C, Ottesen B. Fast track hysterectomy. Eur J Obstet Gynecol Reprod Biol. 2001; 98:18-22. [PubMed: 11516794]

21. American Pain Society. Principles of analgesic use in the treatment of acute pain and cancer pain. 6th. American Pain Society; Glenview (IL): 2008. 
22. Strasberg SM, Linehan DC, Hawkins WG. The accordion severity grading system of surgical complications. Ann Surg. 2009; 250:177-86. [PubMed: 19638919]

23. Melton LJ 3rd. History of the Rochester Epidemiology Project. Mayo Clin Proc. 1996; 71:266-74. [PubMed: 8594285]

24. Aarts MA, Okrainec A, Glicksman A, Pearsall E, Victor JC, McLeod RS. Adoption of enhanced recovery after surgery (ERAS) strategies for colorectal surgery at academic teaching hospitals and impact on total length of hospital stay. Surg Endosc. 2012; 26:442-50. [PubMed: 22011937]

25. Delaney CP, Zutshi M, Senagore AJ, Remzi FH, Hammel J, Fazio VW. Prospective, randomized, controlled trial between a pathway of controlled rehabilitation with early ambulation and diet and traditional postoperative care after laparotomy and intestinal resection. Dis Colon Rectum. 2003; 46:851-9. [PubMed: 12847356]

26. Kehlet H, Mogensen T. Hospital stay of 2 days after open sigmoidectomy with a multimodal rehabilitation programme. Br J Surg. 1999; 86:227-30. [PubMed: 10100792]

27. Maessen J, Dejong CH, Hausel J, Nygren J, Lassen K, Andersen J, et al. A protocol is not enough to implement an enhanced recovery programme for colorectal resection. Br J Surg. 2007; 94:22431. [PubMed: 17205493]

28. Holte K, Sharrock NE, Kehlet H. Pathophysiology and clinical implications of perioperative fluid excess. Br J Anaesth. 2002; 89:622-32. [PubMed: 12393365]

29. Lobo DN, Bostock KA, Neal KR, Perkins AC, Rowlands BJ, Allison SP. Effect of salt and water balance on recovery of gastrointestinal function after elective colonic resection: a randomised controlled trial. Lancet. 2002; 359:1812-8. [PubMed: 12044376]

30. Dudley HF, Boling EA, Lequesne LP, Moore FD. Studies on antidiuresis in surgery: effects of anesthesia, surgery and posterior pituitary antidiuretic hormone on water metabolism in man. Ann Surg. 1954; 140:354-67. [PubMed: 13198073]

31. Srinivasa S, Hill AG. Perioperative fluid administration: historical highlights and implications for practice. Ann Surg. 2012; 256:1113-8. [PubMed: 22824855]

32. Charoenkwan K, Phillipson G, Vutyavanich T. Early versus delayed (traditional) oral fluids and food for reducing complications after major abdominal gynaecologic surgery. The Cochrane Database of Systematic Reviews. 2007; (Issue 4) Art. No.: CD004508. DOI: 10.1002/14651858.CD004508.pub3.

33. Levy BF, Scott MJ, Fawcett W, Fry C, Rockall TA. Randomized clinical trial of epidural, spinal or patient-controlled analgesia for patients undergoing laparoscopic colorectal surgery. Br J Surg. 2011; 98:1068-78. [PubMed: 21590762]

34. Sprung J, Sanders MS, Warner ME, Gebhart JB, Stanhope CR, Jankowski CJ, et al. Pain relief and functional status after vaginal hysterectomy: intrathecal versus general anesthesia. Can J Anaesth. 2006; 53:690-700. [PubMed: 16803917] 


\section{Box 1}

\section{Enhanced Recovery Pathway}

I. Preoperative diet vomiting prophylaxis

Fluid balance

Analgesia
Anesthesia in pelvic
organ
prolapse surgery

III. Postoperative

Activity

Diet

Analgesia
- Evening before surgery: carbohydrate loading drink; may eat until midnight

- May ingest fluids up to $4 \mathrm{~h}$ before procedure

- Eliminate use of mechanical bowel preparation; rectal enemas still performed

- Celecoxib $400 \mathrm{mg}$ orally once

- Acetaminophen $1,000 \mathrm{mg}$ orally once

- Gabapentin $600 \mathrm{mg}$ orally once

- Before incision ( $\pm 30 \mathrm{~min}$ ): dexamethasone $4 \mathrm{mg}$ IV once plus droperidol $0.625 \mathrm{mg}$ IV once

- Before incision closure ( $\pm 30 \mathrm{~min})$ : granisetron $0.1 \mathrm{mg} \mathrm{IV}$ once

- Goal: maintain intraoperative euvolemia

D Decrease crystalloid administration

$O$ Increase colloid administration if needed

- Opioids IV at discretion of anesthesiologist supplemented with ketamine, ketorolac, or both

- After incision closure: injection of bupivacaine at incision site

- Subarachnoid block containing bupivacaine and hydromorphone (40-100 micrograms)

- Sedation vs "light" general anesthetic at the discretion of the anesthesiologist

- Ketorolac $15 \mathrm{mg}$ IV at the end of the procedure for patients able to tolerate it

- No wound infiltration with bupivacaine in this cohort

- Evening of surgery: out of bed greater than $2 \mathrm{~h}$, including one or more walks and sitting in chair

- Day after surgery and until discharge: out of bed greater than $8 \mathrm{~h}$ including four or more walks and sitting in chair

- Patient up in chair for all meals

- No nasogastric tube; if nasogastric tube used intraoperatively, remove at extubation

- Patient encouraged to start low residual diet $4 \mathrm{~h}$ after procedure

- Day of surgery: one box of liquid nutritional supplement; encourage oral intake of at least $800 \mathrm{~mL}$ of fluid, but no more than $2,000 \mathrm{~mL}$ by midnight

- Day after surgery until discharge: two boxes of liquid nutritional supplement; encourage daily oral intake of $1,500-2,500 \mathrm{~mL}$ of fluids

- Osmotic diarrhetics: senna and docusate sodium; magnesium oxide; magnesium hydroxide as needed

- Goal: no IV patient-controlled analgesia

- Oral opioids

Oxycodone $5-10 \mathrm{mg}$ orally every $4 \mathrm{~h}$ as needed for pain rated 4 or greater or greater than patient stated comfort goal ( $5 \mathrm{mg}$ for pain rated $4-6$ or $10 \mathrm{mg}$ for pain rated 7-10); for patients who received intrathecal analgesia, start $24 \mathrm{~h}$ after intrathecal dose given

- Scheduled acetaminophen*

O Acetaminophen $1,000 \mathrm{mg}$ orally every $6 \mathrm{~h}$ for patients with no or mild hepatic disease; acetaminophen $1,000 \mathrm{mg}$ orally twice daily for patients with moderate hepatic disease; maximum acetaminophen should not exceed $4,000 \mathrm{mg} / 24 \mathrm{~h}$ from all sources 
- $\quad$ Scheduled NSAIDs

O Ketorolac $15 \mathrm{mg}$ IV every $6 \mathrm{~h}$ for four doses (start no sooner than $6 \mathrm{~h}$ after last intraoperative dose); then, ibuprofen $800 \mathrm{mg}$ orally every $6 \mathrm{~h}$ (start $6 \mathrm{~h}$ after last ketorolac dose administered)

- If patient unable to take NSAIDs

Tramadol $100 \mathrm{mg}$ orally 4 times a day (start at 6:00 am day after surgery) for patients younger than 65 years of age and no history of renal impairment or hepatic disease; tramadol $100 \mathrm{mg}$ orally twice daily (start at 6:00 am day after surgery) for patients 65 years of age or older or creatinine clearance less than $30 \mathrm{~mL} / \mathrm{min}$ or history of hepatic disease

- Breakthrough pain (pain greater than 7 more than $1 \mathrm{~h}$ after receiving oxycodone)

O Hydromorphone $0.4 \mathrm{mg}$ IV once if patient did not receive intrathecal medications; may repeat once after $20 \mathrm{~min}$ if first dose ineffective

- IV patient-controlled analgesia

O Hydromorphone patient-controlled analgesia started only if continued pain despite 2 doses of IV hydromorphone

Fluid balance

- Operating room fluids discontinued on arrival to floor

- Fluids at $40 \mathrm{~mL} / \mathrm{h}$ until 8:00 am on day after surgery and then discontinued

- Peripheral lock IV when patient had $600 \mathrm{~mL}$ orally intake or at 8:00 am on day after surgery, whichever came first

IV, intravenous; NSAIDs, nonsteroidal anti-inflammatory drugs.

Doses for patients greater than $80 \mathrm{~kg}$ and younger than 65 years of age; doses adjusted as appropriate for patients less than $80 \mathrm{~kg}$ or 65 years of age or older. 


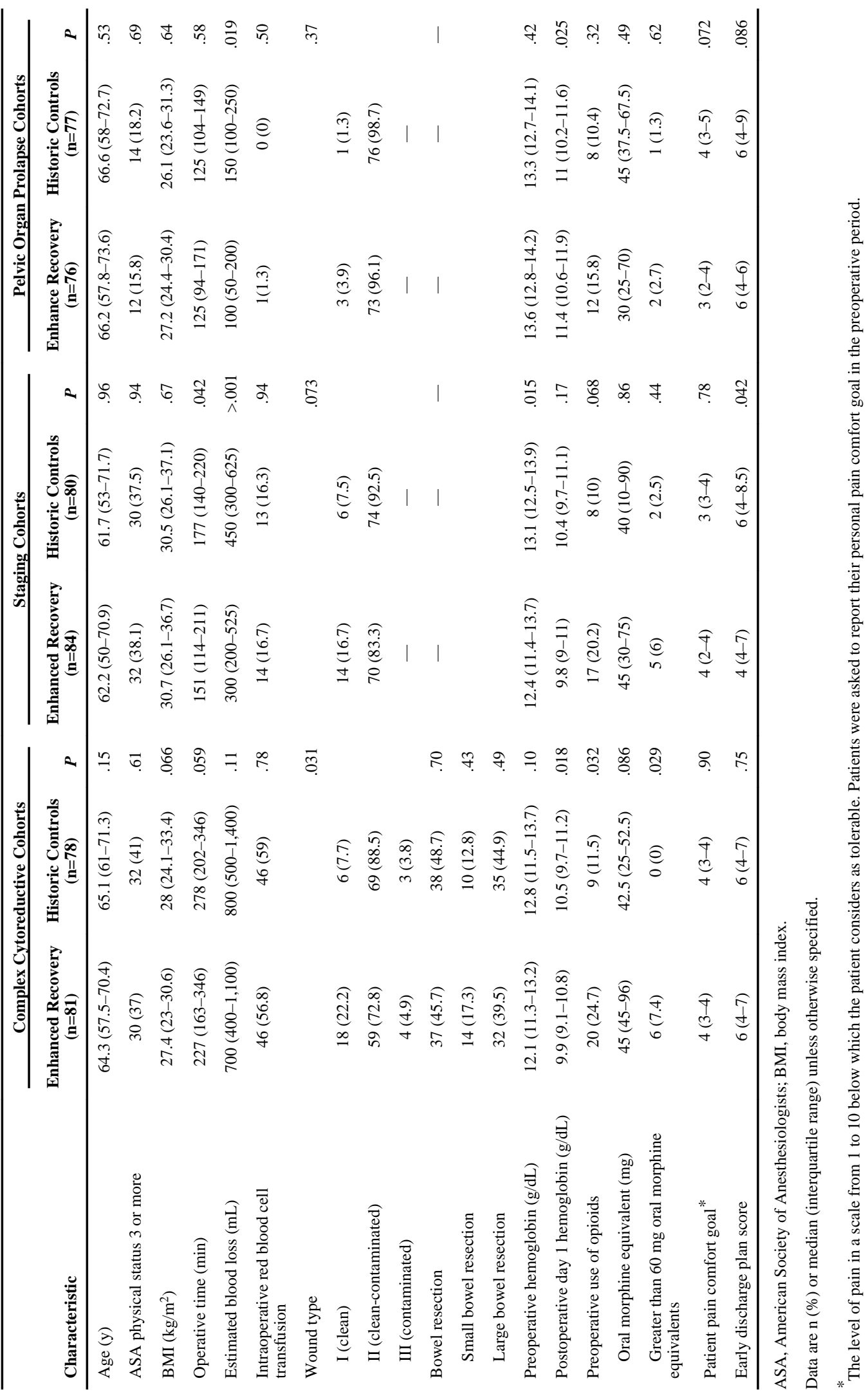


Table 2

Comparison of 30-Day Costs Between Enhanced Recovery Cases and Controls

\begin{tabular}{lccc}
\hline & Enhanced Recovery & Historic Controls & $\boldsymbol{P}$ \\
\hline Complex cytoreductive & $(\mathrm{n}=81)$ & $(\mathrm{n}=78)$ & .006 \\
Mean & $\$ 33,106.24$ & $\$ 40,748.57$ & \\
Median & $\$ 27,129.20$ & $\$ 33,762.51$ & \\
Interquartile range & $(22,950.24-39,188.39)$ & $(26,845.57-42,257.90)$ & \\
Staging & $(\mathrm{n}=84)$ & $(\mathrm{n}=80)$ & .13 \\
Mean & $\$ 22,482.00$ & $\$ 25,757.48$ & \\
Median & $\$ 21,046.76$ & $\$ 22,150.67$ & \\
Interquartile range & $(16,544.71-26,542.04)$ & $(18,541.56-26,594.77)$ & \\
Pelvic organ prolapse & $(\mathrm{n}=76)$ & $(\mathrm{n}=77)$ & .056 \\
Mean & $\$ 10,547.87$ & $\$ 10,989.08$ & \\
Median & $\$ 9,657.45$ & $\$ 10,354.74$ & \\
Interquartile range & $(7,938.1-11,541.22)$ & $(8,495.37-11,470.75)$ & \\
\hline
\end{tabular}




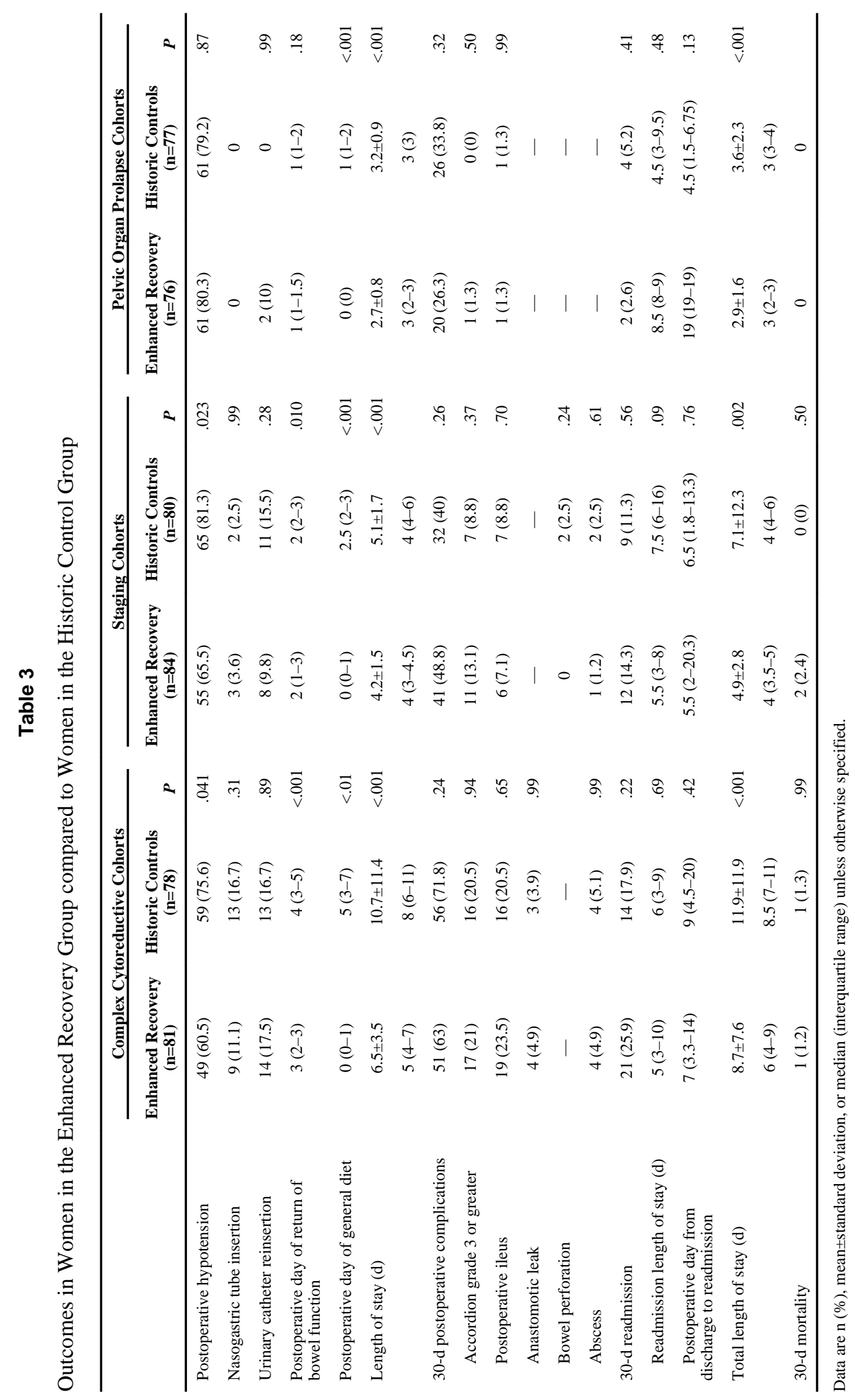

